\title{
Research on Fuzzy Control of Electrical Heater Based on PLC
}

\author{
Chao Yang ${ }^{1, a}$, Chang Su ${ }^{1, b}$, Cong Tian ${ }^{1, c}$ \\ ${ }^{1}$ Heilongjiang University of Technology, Jixi, Heilongjiang, China, 158100 \\ a email jxdxyc@163.com, bemail jxdxsc@163.com, email zwqkt@126.com
}

Keywords: Fuzzy Control, Electrical Heater, PLC

\begin{abstract}
Temperature is a very popular parameter in flow industry, so temperature control is an emphasis of process control. Fuzzy control is an important application filiation of fuzzy mathematics based on the theory fuzzy sets. This paper firstly analyzes the basic principles of fuzzy control, the structure selection of fuzzy controller and the design of fuzzy controller. Then, it explores the process of realization of fuzzy control based on PLC to provide some references for the relevant researchers.
\end{abstract}

\section{Introduction}

In the actual industrial process control system, it involves a lot of nonlinear, large time lag complex system, which makes it very difficult to establish the mathematical model, or it is impossible to model. In recent years, many new control methods have been put forward to deal with this kind of problem, and fuzzy control is one of them. Using fuzzy logic to control the experience of people summed up as a set of conditional statements with a qualitative description, then using fuzzy set theory, the quantitative, the controller can receive the person's experience, imitate the operation strategy, which formed the so-called fuzzy controller. This controller from the experience of the operator or the actual experimental results, rather than from the mathematical model, the manual operation can completely replace the uncertainty can be overcome, so that the system has higher reliability. More importantly, it can solve many problems which are difficult to be solved by the linear system theory. It is generally believed that the fuzzy control is a kind of control method which has better control effect for the system with delay and random disturbance. In the field of application of fuzzy control technology, the fuzzy controller is basically realized by personal computer, single chip microcomputer or special fuzzy control chip. In industrial control, programmable logic controller application is extremely wide, if the fuzzy control technology combined with PLC, using PLC to realize fuzzy controller, the fuzzy control will play a greater role in the industrial control. Now there are some PLC manufacturers have fuzzy control module for users to choose, but the price is very expensive. At present, in industrial applications, especially in process control, the programmable logic controller, if hardware based conventional control system, realized fuzzy controller using fuzzy control software programming, will reduce the cost of the system. At the same time, it has created the conditions for using the fuzzy control technology to transform the original conventional control method.

\section{Strategy of Fuzzy Control}

Basic Principles of Fuzzy Control. The input of the fuzzy PID control system is the deviation e and the variation rate of the deviation ec, the output is $\mathrm{K}_{\mathrm{P}}, \mathrm{K}_{\mathrm{I}}$, and $\mathrm{K}_{\mathrm{D}}$. The system mainly consists of two parts, which are fuzzy controller and PID controller. In working condition, collect the object temperature, the measured value of $\mathrm{T}(\mathrm{k})$ and $\mathrm{T}^{*}$ and given the value of e and ec, respectively, 
multiplied by the quantization factor after each fuzzy reasoning, output $\mathrm{K}_{\mathrm{P}}, \mathrm{K}_{\mathrm{I}}$, and $\mathrm{K}_{\mathrm{D}}$ parameter tuning, complete control of the object. Can be widely used in such aspects as temperature control, flow control, motor speed control. The program will be tested in the temperature control. First, we get an accurate measurement of variable from e, according to the relationship of the fuzzy membership of fuzzy vector E, and then, according to the fuzzy relation determined by the membership function of fuzzy rules and input the actual experience of manual control model based on fuzzy vector and the output vector of the fuzzy theory to make fuzzy inference. One of the most critical steps is to summarize the experience of the operator into some rules, through fuzzy mathematics processing and storage to the computer, the formation of fuzzy control rules, and follow the fuzzy reasoning process of human brain, to determine the rule of inference.

Structure Selection of Fuzzy Controller. According to the characteristics of the controlled object, the fuzzy controller according to the structure is divided into a single input single output and multiple input multiple outputs (MIMO) two. As for some complex MIMO system is difficult to directly extract the control rules, so the method is commonly used in the MISO structure, and then use the 5150 design methods of designing the fuzzy controller, fuzzy multi variable control system decoupling. The design of fuzzy controller for the system is discussed in this study. Single variable fuzzy control system, usually the single variable fuzzy controller the number of input variables is called dimension fuzzy controller based on this, the general can be divided into one-dimensional single variable fuzzy controller fuzzy controller, fuzzy controller, fuzzy controller. Electric heater is the most commonly used equipment in process industry, and its temperature control is a key point of process control. The electric heater has large time delay, nonlinear and difficult to establish accurate mathematical model and so on. Therefore, the fuzzy control strategy is used to control the system. In general, a two-dimensional fuzzy controller in the control process not only on the feedback error of the controlled variable, but also on the feedback error change rate of the controlled variable, so as to ensure the stability of the system, and will not produce the oscillation phenomenon is the industrial application is ideal, is the most commonly used controller, so the following mainly for two dimension fuzzy controller the specific design method is given.

\section{Design of Fuzzy Controller. T}

The system uses two inputs and three outputs fuzzy controller. The input variables of the controller are temperature deviation e and deviation change rate ec are calculated by the following formulas:

$$
\begin{aligned}
& e(k)=T(k)-T^{*} \\
& e c(k)=\frac{e(k)-e(k-1)}{t}
\end{aligned}
$$

Among the above formulas, $T^{*}$ is the given temperature value; $t$ is the sampling period. According to the principle of PID parameter, we establish the inference rule. The inference rule of $\mathrm{KP}$ is shown in Table 1. 
Table 1. Inference rule of KP

\begin{tabular}{|c|c|c|c|c|c|c|c|}
\hline \multirow{2}{*}{ KP } & \multicolumn{7}{|c|}{ ec } \\
\cline { 2 - 8 } & PB & NB & NM & NS & ZO & PS & PM \\
\hline NB & PB & PB & PB & ZM & PS & ZO & ZO \\
\hline NM & PB & PB & PM & PS & PS & ZO & NS \\
\hline NS & PM & PM & PM & PS & ZO & NS & NS \\
\hline ZO & PM & PM & PS & ZO & NS & NM & NM \\
\hline PS & PS & PS & ZO & NS & NS & NM & NM \\
\hline PM & PS & ZO & NS & NM & NM & NM & NB \\
\hline PB & ZO & ZO & NM & NM & NB & NB & NB \\
\hline
\end{tabular}

Then, we use the function of evalfis to calculate the table of fuzzy control. The program is as follows:

$$
\begin{aligned}
& \text { List =zeros( 13, 13, 3); } \\
& \text { for } i=1: 13 \\
& \text { for } \mathrm{j}=1: 13 \\
& e(i)=-7+i \text {; } \\
& \text { ec }(j)=-7+j \text {; } \\
& \operatorname{list}(\mathrm{i}, \mathrm{j}, \text { : ) =evalfis([e(i), e(j)], fuzzy); }
\end{aligned}
$$

end

end

list=ceil(list)

\section{Realization of Fuzzy Control Based on PLC}

Program Design. Based on the SIEMENS STEP7 as a software programming environment, as far as possible the use of method of modular programming, in the software design process of the complete, according to the diagram and design the detailed function control program is part of the core of the whole work. Using MPI network communication between PC and PLC connected close communication in this study, the preparation of SETP7 program in the host computer, to achieve the PLC program through the MPI network. In order to the main program cycle, that is, the organization block OBI, concise and reasonable, the fuzzy query table query program using functional block nested program design method. Define a function block FB50, used to realize the fuzzy query function, in the OBI can call. Offset stored as 0 of the query program. The inputs of the fuzzy quantization procedure shows that the storage of E Mw22 quantitative value stored in MW32 is EC, the quantization value, first determine the line number, namely the use of MW22 on the quantitative range of values are compared to determine the corresponding operation output row, then activate the corresponding row column lookup procedure, namely column elements on the corresponding line of the comparison operation to determine the coincidence in the two-dimensional assignment control. Each row in the query program after the implementation of a conditional jump action, if a store of storage of electricity on M50\#0, the program for the jump, the control output assignment procedure. This jump action significantly reduces the PLC query time. 
Operation Results. The STEP7 software programming work and WinCC software configuration work together, achieve a complete set of fuzzy control system of experimental electric heater PCT process control experimental device based on the development of. By experiment we run the test without relying on accurate mathematical model under the condition of application of fuzzy control strategy for nonlinear, delay electric heater temperature control and the feasibility of object specific control effect, and use PLC to realize the feasibility and applicability of fuzzy control by software programming method. The results comparison of fuzzy control and traditional control are shown in Table 2.

Table 2.Results Comparison of Fuzzy Control and Traditional Control

\begin{tabular}{|c|c|c|}
\hline Control Strategy & Overshoot & Adjusting Time \\
\hline Fuzzy Control & $3^{\circ} \mathrm{C}$ & $110 \mathrm{~s}$ \\
\hline Traditional Control & $10^{\circ} \mathrm{C}$ & $220 \mathrm{~s}$ \\
\hline
\end{tabular}

Compared with the traditional PID control, the response of fuzzy control has a smaller overshoot, and the adjusting time is relatively short, depend on the object model is low, its quality control was significantly higher than the control performance index of traditional PDI, but also should be noted that although the fuzzy control in the dynamic response and internal inhibition the change of system parameters on the system output effect can achieve better effect. The control strategy which relies on expert experience is actually equivalent to a PD controller. Its design has a strong subjectivity, so simple fuzzy control although the structure is simple. There is a lack of performance in the steady state, as shown in the graph in response, the control effect will appear steady vibration slightly, it needs further to the fuzzy control and Pm control strategy and other combination of design and engineering fuzzy integrated control system used in the realization of the. But overall, based on the research process does not depend on the accurate model of the object, using PLC to realize fuzzy control strategy has achieved a better control of temperature, the experimental demonstration for the applicability of fuzzy control process of similar objects, and gives the concrete realization method of PLC programming reference.

\section{Conclusion}

Fuzzy control is a suitable method to solve the time-delay and nonlinear problem when it is difficult to establish accurate mathematical model of the control. PLC has the characteristics of reliable operation, simple operation and flexible development. It can be easily realized by software of fuzzy control strategy. This paper not only studies the temperature control problem, but also provides an ideal temperature control scheme for some similar industrial control occasions.

\section{References}

[1] Qu Feng, Xu Zhonghong, Sun Ji, Journal of Nanjing Normal University (Engineering and Technology Edition), Vol. 7(2007) No 4, p.23-27

[2] Liu Zenghuai, Wang Lizhen, He Guangxiang, Automation \& Instrumentation, Vol. 31(2011) No 10 , p.27-30

[3] Feng Bo, Xu Shuhua, Journal of Qingdao University (Engineering \& Technology Edition), Vol. 26(2011) No 1, p.14-18

[4] Zhang Yu, Li Ming, Tang Zhibiao, Zhang Hongxing, Journal of Nanchang University (Engineering \& Technology), Vol. 27(2005) No 3, p.59-63 